\title{
Evaluating Integrity and Continuity Over Time in Advanced RAIM
}

\author{
Carl Milner \\ ENAC \\ Toulouse, France \\ milner@recherche.enac.fr
}

\author{
Boris Pervan \\ Illinois Institute of Technology \\ Chicago, IL \\ pervan@iit.edu
}

\author{
Juan Blanch \\ Stanford University \\ Stanford, CA \\ blanch@stanford.edu
}

\author{
Mathieu Joerger \\ Virginia Tech \\ Blacksburg, VA \\ joerger@vt.edu
}

\begin{abstract}
The Service Evolutions Sub-Group (SESG) of Working Group C, the joint technical committee formed from U.S and E.U public bodies for the purpose of promoting GPS-Galileo applications, has spent the past years working on a number of topics relating to Advanced RAIM development and its early stages of standardization. This paper addresses the mapping of integrity and continuity requirements from the operational (i.e. per hour) to the algorithmic (per sample). It provides a method to rigorously account for the impact on integrity risk of multiple exposures to hazardously misleading events over time, and for the impact on false alert probability of multiple detection tests over time. This analysis leads to the estimation and bounding of the "number of effectively independent samples", or more concisely the "number of effective samples (NES), for integrity and continuity within their respective exposure windows.
\end{abstract}

Keywords - integity, continuity, Advanced RAIM

\section{INTRODUCTION}

This paper presents a framework for the treatment of the number of effectively independent samples over an exposure window is developed. The paper is split into three parts.

Section II of this paper describes a generic process to determine the number of effective samples without assumptions regarding the temporal correlation properties of the errors and without assumptions regarding the actual implementation of the airborne algorithm. Like RAIM, the initial baseline ARAIM airborne algorithms (e.g. in the ARAIM Milestone 3 report) assumed a single sample, allocating the full continuity and integrity risks required at the operational level to the algorithm at each sample. While this approach is a reasonable approximation for some phases of flight given the observed error margins, it may underestimate the effect of temporal exposure, especially for long exposure times. In Section II, key definitions are given of the critical events, namely that a false detection event occurs when, under the fault-free state, starting at any sample within the exposure window, the false detection condition is true for at least the time to alert (TTA) duration. Similar, a hazardously misleading information (HMI) event occurs when, under the fault hypothesis, starting at any sample within the exposure window, both the no detection condition and the positioning failure condition are true sequentially for the TTA duration. The number of effective samples (NES) is then defined as: (a) for continuity, the ratio of the probability of false alert (FA) during an operation over the probability of FA at a single epoch (b) for integrity, the ratio of the risk of hazardously misleading information (HMI) during an operation over the risk of HMI at a single epoch. Following these definitions, upper bounds on NES are developed.

Section III provides refinements of the NES analysis accounting for the temporal behavior of the error sources and variability of the receiver algorithm implementation. The impact of temporal correlation of the input measurements is addressed through simulation employing a First Order Gauss Markov Process, followed by analysis of a real data collection.

Section IV discusses the consequences of this work for the Airborne Definition Document (ADD) and a performance evaluation is presented.

\section{GENERIC ANALYSIS}

\section{A. Framework and Definitions}

\section{1) Requirements Interpretation}

In order to proceed, it is necessary to clarify our interpretation of the requirements, both at the SARPs level (SARPs stands for ICAO's Standards and Recommended Practices) and at the constellation service provider (CSP) commitments level.

2) $S A R P S$

This section will treat the definitions and statements relating to integrity and continuity as made in the SARPs [8].

Integrity is a measure of the trust that can be placed in the correctness of the information supplied by the total system. Integrity includes the ability of a system to provide timely and valid warnings to the user (alerts).

Continuity is the capability of the system to perform its function without unscheduled interruptions during the intended operation.

Time-To-Alert (TTA) is the maximum allowable time elapsed from the onset of the navigation system being out of tolerance until the equipment enunciates the alert.

We make the following interpretations. Loss of integrity occurs when trust is lost for a period that exceeds TTA within the period of operation (see Figure 1). Loss of continuity occurs when an alert condition persists for a duration exceeding TTA. 
TABLE I. SIGNAL-IN-SPACE INTEGRITY REQUIREMENTS

\begin{tabular}{|l|l|c|c|}
\hline \multirow{2}{*}{ Operation } & \multicolumn{3}{|c|}{ Requirements } \\
\cline { 2 - 4 } & \multicolumn{1}{|c|}{ Integrity } & TTA & Continuity \\
\hline $\begin{array}{l}\text { En-route, } \\
\text { NPA }\end{array}$ & $1-10-7 / \mathrm{h}$ & $10 \mathrm{~s}$ & $1-10-4 / \mathrm{h}$ to $1-10-8 / \mathrm{h}$ \\
\hline Approach & $1-10-7$ in any approach & $6 \mathrm{~s}$ & $1-10-6$ per $15 \mathrm{~s}$ \\
\hline
\end{tabular}

For an approach, the period of operation, here equivalent to the exposure time, is $150 \mathrm{~s}$ for integrity purposes and $15 \mathrm{~s}$ for the final approach segment critical to continuity. For en-route flight and non-precision approach (NPA), we assume that the exposure period for both integrity and continuity is fixed at one hour. We made this assumption considering two possible interpretations of the requirements:

1 - The period of operation is taken to be fixed at one hour, therefore fixing the exposure window over which to assess integrity, or

2 - The requirement expresses a risk rate, such that a variable period of operation can be considered, and the risk requirement is proportional to the exposure window length i.e. for rate $r$ and window length $T$ the risk is then is then $I(T) \approx r \times T$.

Interpretation 1 captures the intent of the requirement in the SARPs Table 3.7.2.4-1. Interpretation 2 becomes impractical when accounting for non-monitored latent faults.

With regard to Interpretation 2, consider the example of a GPS constellation fault, which is assumed a rate of occurrence of 10 $8 / \mathrm{h}$. Firstly, take the case of values of $T$ larger than 1 hour. To evaluate $P_{\text {const }}$, we must account for the probability of the fault being present at the start of the operation or of it occurring during the operation. The first is the product of its rate of occurrence and its mean time to notify or MTTN $(10-8 \times$ MTTN), and the second is the product of the rate of occurrence and the exposure window length $T$. The total probability (10-8) therefore is $10-8(M T T N+T)$. However, as the exposure window increases in length, the ratio of this value and $I(T)$ approaches a constant, since MTTN become insignificant against $T$. Secondly, consider the case of values of $T$ smaller than 1 hour e.g. 0.1 hours. The requirement is then reduced to $I(T)<I(1$ hour $)$ whilst the probability of a GPS constellation fault can never be inferior to $10-8 \times M T T N$. Under this case, it therefore becomes impractical to interpret the requirement using short exposure windows to handle non-monitored faults.

To conclude, it is proposed to fix the exposure window to a practical value of 1 hour thereby adopting Interpretation 1 above. Once the length of the exposure window is determined, then, by definition, the integrity requirement must be met for each and every such exposure window. Loss of integrity occurs throughout the exposure window if a hazardous condition is present without alert for a duration longer than one TTA as illustrated in Figure 1 and Figure 2.

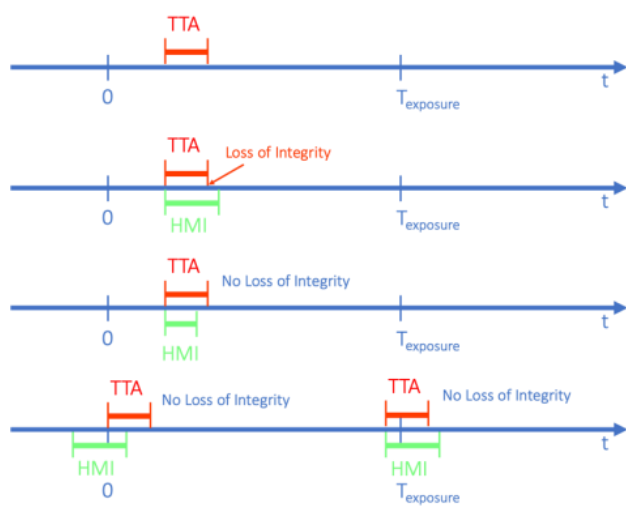

Figure 1: Loss of Integrity

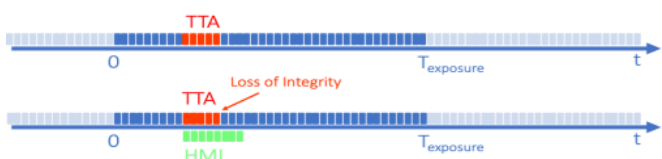

Figure 2: Loss of Integrity - Discrete Time

\section{3) Service Commitments}

a) GPS Service Commitments

The GPS service commitments also provide similar definitions [9] for the SPS SIS Integrity in terms of a Major Service Failure (MSF), expressed as the probability that the SPS SIS's instantaneous URE exceeds the SIS URE NTE tolerance (be 4.42 times the upper bound on the $\left.\sigma_{U R A}\right)$ without a timely alert being issued.

The GPS service commitment for this value is currently 10-5 per satellite per hour, which is employed here as a rate $r_{\text {sat }}$. Commitments are also made on the CSP's mean time to notify (MTTN) of 1 hour [9]. For a fault hypothesis $k$, these quantities may be related to the state probability as follows:

$$
P_{\text {fault }, k} \cong r_{k} \int_{-\infty}^{0} e^{\frac{-t}{T_{m}}} d t \cong r_{k} \times T_{m, k}
$$

where $r_{k}$ is the fault rate and $T_{m, k}$ is the MTTN for fault hypothesis $k$. The rate fault $r_{k}=1 / M T B F$, where MTBF is the Mean Time Between Failure (MTBF). For composite faults, both the rate and MTTN may be derived from their counterparts broadcast within ISM and derived in Appendix C. A more precise computation of the state probability may be given as follows, accounting for MTBF and MTTN:

$$
P_{\text {fault }, k}=\frac{M T T N}{M T B F+M T T N}=\frac{T_{m, k}}{\frac{1}{r_{k}}+T_{m, k}}=\frac{r_{k} T_{m, k}}{1+r_{k} T_{m, k}} \cong r_{k} T_{m, k}
$$

\section{b) Galileo Service Commitments}

Integrity is to be developed in future issues of the Galileo Service Definition Document (SDD) [10].

\section{B. Temporal Parameters}

A number of time intervals must be expressed which relate to the system requirements, receiver requirements and ISM contents [5], [11]. The operation exposure time and required 
TTA are taken from the requirements along with the receiver measurement processing and position, velocity, and time (PVT) estimation output interval. The CSP monitoring MTTN is set here at the default value of GPS, since it is well established, but may differ for other constellations. The exposure timeline is shown in Figure 3. Additional terms are provided in Table II. For analysis purposes, we nondimensionalize time by $T_{a}$. We define: $n \triangleq \frac{T_{e}}{T_{a}}, m \triangleq \frac{T_{m}}{T_{a}}$.

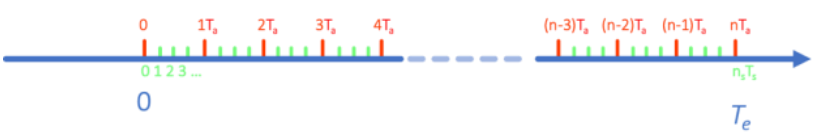

Figure 3: Exposure timeline $T_{e}$

\section{Event Definitions}

The purpose of this paper is to relate continuity and integrity requirements from the operational level to the receiver level.

TABLE II. TEMPORAL PARAMETERS

\begin{tabular}{|c|c|c|c|}
\hline Name & Description & $\begin{array}{l}\text { Value for } \\
\text { RNP }\end{array}$ & Value for LPV \\
\hline$T_{e}$ & Operation Exposure time & $3600 \mathrm{~s}$ & $\begin{array}{l}\text { 150s for } \\
\text { integrity } \\
15 \text { s for } \\
\text { continuity }\end{array}$ \\
\hline$T_{a}$ & Required Time to Alert & $10 \mathrm{~s}$ & $6 s$ \\
\hline$T_{m}$ & $\begin{array}{c}\text { CSP monitor Mean Time To } \\
\text { Notify (MTTN) }\end{array}$ & $\begin{array}{l}\text { 3600s for } \\
\text { GPS }\end{array}$ & 3600 s for GPS \\
\hline$T_{m, k}$ & $\begin{array}{l}\text { Derived MTTN for fault } \\
\text { hypothesis k based on } \\
\text { broadcast parameters (c.f. } \\
\text { Appendix C) }\end{array}$ & & \\
\hline$T_{d}$ & $\begin{array}{l}\text { Receiver fault detection and } \\
\text { exclusion (FDE) interval }\end{array}$ & $0.2 \mathrm{~s}-10 \mathrm{~s}$ & $0.2 \mathrm{~s}-6 \mathrm{~s}$ \\
\hline$T_{S}$ & Receiver PVT output interval & $0.2 \mathrm{~s}-1 \mathrm{~s}$ & $0.2 \mathrm{~s}-1 \mathrm{~s}$ \\
\hline$n$ & $\begin{array}{c}\text { Number of TTA periods per } \\
\text { exposure window }\end{array}$ & 360 & 25 \\
\hline$n_{d}$ & $\begin{array}{c}\text { Number of FDE samples per } \\
\text { exposure window }\end{array}$ & $360-18000$ & $\begin{array}{l}25-750 \text { for } \\
\text { integrity }\end{array}$ \\
\hline$n_{s}$ & $\begin{array}{c}\text { Number of PVT samples per } \\
\text { exposure window }\end{array}$ & $3600-18000$ & $\begin{array}{l}150-750 \text { for } \\
\text { integrity }\end{array}$ \\
\hline$n_{d a}$ & $\begin{array}{l}\text { Number of FDE samples per } \\
\text { TTA }\end{array}$ & $1-50$ & $1-30$ \\
\hline$n_{s a}$ & $\begin{array}{l}\text { Number of PVT samples per } \\
\text { TTA }\end{array}$ & $10-50$ & $6-30$ \\
\hline$m$ & $\begin{array}{l}\text { Number of TTA periods per } \\
\text { MTTN }\end{array}$ & 360 & 25 \\
\hline$\tau$ & $\begin{array}{l}\text { Code-Carrier smoothing time } \\
\text { constant }\end{array}$ & $100 \mathrm{~s}$ & $100 \mathrm{~s}$ \\
\hline
\end{tabular}

Fault-Free Hypothesis: The condition of no satellite or constellation fault being present, denoted by $H_{0}$ and by $H_{0, i}$ for specifically at epoch $i$

False Alert (FA): The detection of a fault under the fault-free hypothesis $H_{0}$.

False Alert (FA) event: Sequential FA over all $n_{d a}$ FDE samples within a period of length $T_{a}$ given $H_{0}$.
A receiver may meet the above 'FA event' definition by only alerting if the FA condition remains true for the $T_{a}$ duration.

$P_{f a}$ : Probability of any FA event over an exposure interval $T_{e}$

$$
P_{f a}\left(n_{d}, H_{0}\right)=P\left\{\bigcup_{j=1}^{n_{d}-n_{d a}} \bigcap_{i=j}^{j+n_{d a}}\left(F A_{i}, H_{0, i}\right)\right\}
$$

The following terms relate to the integrity:

Fault Hypothesis $k$ : The condition of a fault event, indexed by $k$, following the airborne algorithm function, denoted by $H_{k}$ and by $H_{k, i}$ for specifically at epoch $i$

Positioning Failure $(P F)$ : The presence of a positioning error exceeding the protection level.

Positioning Failure (PF) event: Sequential PF over all $n_{s a}$ PVT samples within a period of length $T_{a}$.

No Detection $(N D)$ : The condition that no monitor test statistic exceeds its respective threshold at a single sample $i$.

No Detection (ND) event: Sequential ND conditions over all FDE samples within a period of length $T_{a}$.

Hazardously Misleading Information (HMI): The joint presence of both PF and ND conditions.

For cases where $n_{d}<n_{s}$, the most recent ND condition is paired with the PF condition.

Hazardously Misleading Information (HMI) event: Sequential HMI over all samples within $T_{a}$ given fault hypothesis $H_{k}$.

$P_{h m i}$ : Probability of any HMI event over the exposure interval $T_{e}$.

$$
P_{h m i}\left(n_{s}, H_{k}\right)=P\left\{\bigcup_{j=1}^{n_{s}-n_{s a}} \bigcap_{i=j}^{j+n_{s a}}\left(H M I_{i}, H_{k, i}\right)\right\}
$$

The above definitions given in (3) and (4) express the operational safety metrics employing time indices depicted in Figure 4 . The metrics evaluated by the airborne algorithm are expressed in terms of risk at a single sample, $P_{f a}\left(1, H_{0,1}\right)$ and $P_{h m i}\left(1, H_{k, 1}\right)$ respectively. This leads to the following definitions for the effective number of samples:

$$
\begin{aligned}
& n_{e s, f a}\left(n_{d}\right)=\frac{P_{f a}\left(n_{d}, H_{0}\right)}{P_{f a}\left(1, H_{0,1}\right)} \\
& n_{e s, h m i, k}\left(n_{s} ; n_{d}\right)=\frac{P_{h m i}\left(n_{s}, H_{k} ; n_{d}\right)}{P_{h m i}\left(1, H_{k, 1}\right)}
\end{aligned}
$$

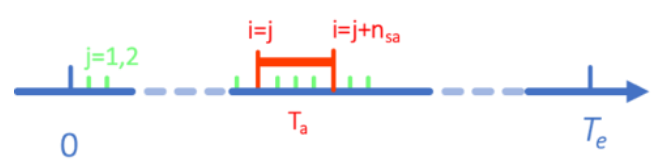

Figure 4: Indices $i$ and $j$ 
Note that a weak assumption that the geometry is unchanging over the exposure interval is used and that it does not negate the validity of the upper bound derived below.

\section{Upper Bounds on the HMI and FA Risks over an Exposure Period}

Given these definitions, the maximum allowable interval between ARAIM monitor tests is $T_{a}$. If fault detection is employed only once per $T_{a}$, such that the number of fault detection epochs $n_{d}$ is equal to the number of TTA periods $n$, then it is not possible for a FA or an HMI event to happen between successive $T_{a}$ intervals. The monitor may sample at a faster rate, for example at intervals $T_{s}$, but it is not required to do so. Therefore, in this section, it is assumed that the fault detection function operates at the minimum rate, which is conservative with respect to integrity performance. This simplifies the subsequent analysis, and it is the reason for the normalization of the other time intervals by $T_{a}$ as performed above.

Using this simplification, an upper bound on the number of effective samples $n_{e s, h m i, k}$ as defined in (4) can be obtained:

$$
P_{h m i}\left(n, H_{k}\right)=P\left(\bigcup_{j=1}^{j=n}\left(H M I_{j}, H_{k, j}\right)\right) \leq \sum_{j=1}^{j=n} P\left(H M I_{j}, H_{k, j}\right)
$$

The above equation shows that it is conservative to assume $n_{e s, h m i, k}=n$. One way to see this is by assuming that $P\left(H M I_{j}, H_{k, j}\right)=P_{h m i}\left(1, H_{k, 1}\right) \quad$ (i.e. assuming that $P\left(H M I_{j}, H_{k, j}\right)$ is independent of time):

$$
P_{h m i}\left(n, H_{k}\right) \leq \sum_{j=1}^{j=n} P\left(H M I_{j}, H_{k, j}\right) \leq n P_{h m i}\left(n, H_{k, 1}\right)
$$

And therefore:

$$
n_{e s, h m i, k}\left(n, H_{k}\right)=\frac{P_{h m i}\left(n, H_{k}\right)}{P_{h m i}\left(1, H_{k, 1}\right)} \leq n=\frac{T_{e}}{T_{a}}
$$

This bound is valid for both the monitored and un-monitored fault hypotheses. In ARAIM, the majority of fault modes have low prior probabilities of occurrence and, therefore, do not need to be monitored, in which case $P_{h m i \mid H_{k}}=1$. Further details may be found in [5], [12]. As seen below, a finer bound exists for the non-monitored hypotheses. Note that the exposure intervals $T_{e}$ may be different for integrity (HMI) and continuity (FA). Further details and rigorous developments are described in Appendices A and B.

The following results are presented as a function of the conditional probability of HMI, $P_{h m i \mid H_{k}}$, or the probability of missed detection. Unlike in RAIM, where $P_{h m i \mid H_{k}}$ is assigned a fixed requirement of 10-3, in ARAIM, the integrity risk can be allocated dynamically at each sample as a function of the ISM, geometry and error models. $P_{h m i \mid H_{k}}$ values may range from $10-5$ to 1 (the value of 1 is used for unmonitored hypotheses).
The results for RNP 0.1 and 0.3 , with $T_{a}=10 \mathrm{sec}$ and $T_{m}=1$ hour, are shown in Figure 5 for a range of $P_{h m i \mid H_{k}}$. The vertical axis represents the number of effectively independent samples for HMI as a function of the exposure time. Figure 6 shows the results for $T_{e}=1$ hour as a function of $P_{h m i \mid H_{k}}$. The number of effectively independent samples for HMI ranges from 2 for unmonitored faults to 360, which applies for monitored faults. Figure 7 shows the corresponding results for LPV 200, with $T_{e}=150 \mathrm{sec}$ and $T_{a}=6 \mathrm{sec}$. Here, the number of effectively independent samples for integrity ranges from 1 for unmonitored faults to 25 for monitored faults.

RNP $0.1 / 0.3$

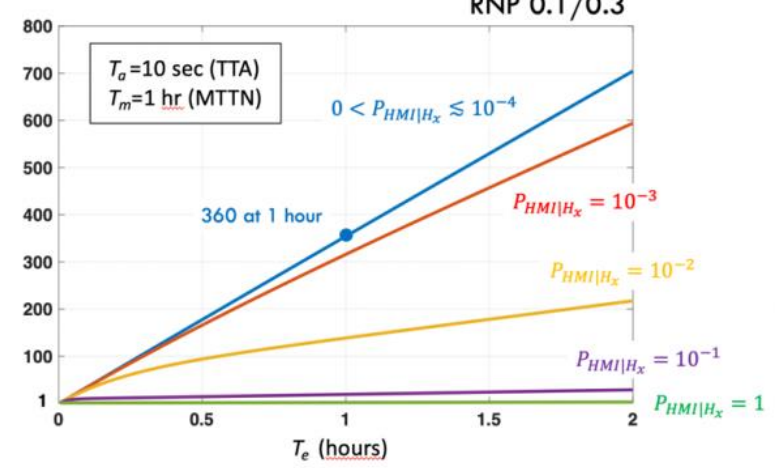

Figure 5: Number of effectively independent samples for integrity vs. $T_{e}$ for RNP $0.1 / 0.3$

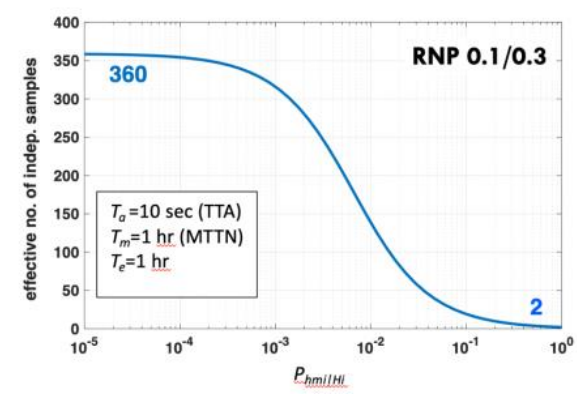

Figure 5: Number of effectively independent samples for integrity vs. $P_{h m i \mid H_{k}}$ for RNP $0.1 / 0.3$, for $T_{e}=1 \mathrm{~h}$

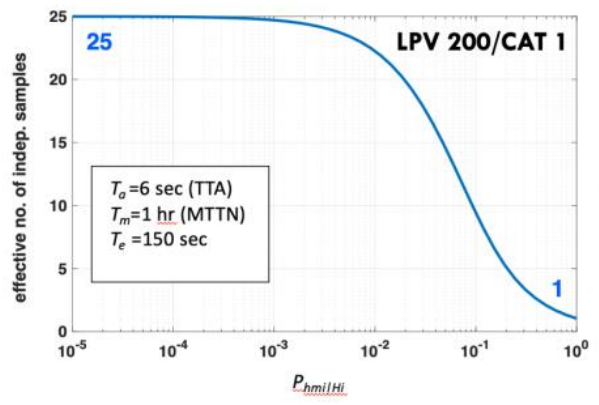

Figure 7: Number of effectively independent samples for integrity vs. $P_{h m i \mid H_{k}}$ for LPV 200, for $T_{e}=150 \mathrm{sec}$

Therefore, for the monitored modes during H-ARAIM RNP 0.3 and RNP 0.1 operations:

$$
\begin{aligned}
& n_{e s, f a}\left(n_{d}\right)<(360 R N P) \text { or }(25 L P V) \\
& n_{e s, h m i}\left(n_{s}\right)<(360 R N P) \text { or }(25 L P V)
\end{aligned}
$$


In the case of the non-monitored modes, the effective number of samples has a tighter upper bound given by:

$$
n_{e s, h m i, k}\left(n_{s}\right)<1+\frac{T_{e}}{T_{m, k}}
$$

This is a consequence of the fact that the probability of having a fault within the exposure window is given by (see Figure 8):

$$
\begin{aligned}
& P_{h m i}\left(n_{s}, H_{k}\right)=r_{k}\left(T_{m, k}+T_{e}\right)= \\
= & r_{k} T_{m, k}+r_{k} T_{e}=r_{k} T_{m, k}\left(1+\frac{T_{e}}{T_{m, k}}\right)
\end{aligned}
$$

Note that the $T_{m, k}$ are derived from the broadcast ISM parameters as described in Appendix C.

Note that (12) grows as, $T_{m, k}$, the MTTN, decreases but is offset by the single epoch probability, as $r_{k} T_{m, k}$ is also scaled appropriately.

$$
\begin{aligned}
P_{h m i}\left(n_{s}, H_{k}\right) & =n_{e s, h m i, k}\left(n_{s}\right) P_{h m i}\left(1, H_{k, 1}\right) \\
= & \left(1+\frac{T_{e}}{T_{m, k}}\right) r_{k} T_{m, k} \cong r_{k} T_{e}
\end{aligned}
$$

In the opposing case, as $T_{m, k}$ grows, the risk increases linearly for $r_{k} T_{m, k} \ll 1$. So for large $T_{m, k}$ :

$$
\begin{gathered}
P_{h m i}\left(n_{s}, H_{k}\right)=n_{e s, h m i, k}\left(n_{s}\right) P_{h m i}\left(1, H_{k, 1}\right)=(1+ \\
\left.\frac{T_{e}}{T_{m, k}}\right) r_{k} T_{m, k} \cong r_{k} T_{m, k}
\end{gathered}
$$

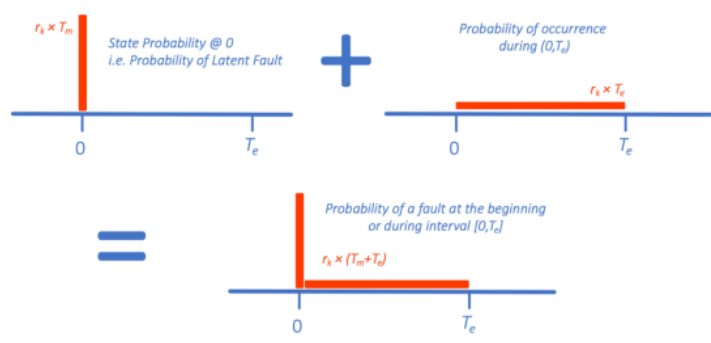

Figure 8: Sum of latent fault and fault during exposure interval

\section{SPECIFIC ANALYSIS}

\section{A. Introduction}

In Section II, an upper bound was given regardless of the receiver implementation and error models. Only assumptions regarding the provision of the ISM and basic elements of the ARAIM design were made. This section includes error modelling for a more precise analysis, beginning with a treatment of the Gauss Markov Process (GMP).

\section{B. The Gauss Markov Process (GMP)}

The standard assumption regarding the temporal behaviour of error sources in GNSS is the first-order Gauss Markov Process [9]. The GMP is parameterised by the process variance $\sigma^{2}$ and its time constant $\tau$ (the reciprocal time constant is $\beta$ ). The autocorrelation function of the GMP is shown in Figure 9:

$$
R(\Delta t)=\sigma^{2} e^{-\beta \Delta t}
$$

$$
\beta=\frac{1}{\tau}
$$

For a given $\Delta t$, the alpha parameter may also be defined as:

$$
\alpha=\frac{R(\Delta t)}{R(0)}=e^{-\beta \Delta t}
$$

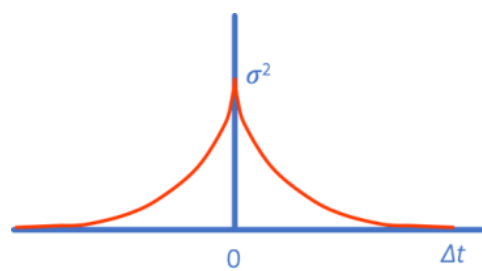

Figure 9: Gauss Markov Process Autocorrelation

Discrete GMP samples of unit variance may be generated by.

$$
x_{i+1}=e^{-\beta T} x_{i}+w_{i}
$$

where:

$x_{0} \quad$ is the initial value following a standard Gaussian distribution

$x_{i} \quad$ is a GMP variable at epoch $i$

$w_{i} \quad$ is a white noise sequence with variance $\left(1-e^{-2 \beta \Delta t}\right)$

$\beta \quad$ is the reciprocal time constant of the process

$T \quad$ is a time interval between adjacent two $x$ 's

ARAIM's ionosphere-free smoothed pseudorange error model contains three terms relating to the Signal-In-Space (SIS), tropospheric and user multipath and noise errors. The autocorrelation function is then:

$$
\begin{gathered}
e_{\text {uere }}=e_{\text {sis }}+e_{\text {trp }}+e_{\text {usr }} \\
R_{\text {uere }}(\Delta t)=\frac{R_{\text {sis }}(\Delta t)+R_{\operatorname{trp}}(\Delta t)+R_{\text {trp }}(\Delta t)}{R_{\text {sis }}(0)+R_{\text {trp }}(0)+R_{\text {trp }}(0)}
\end{gathered}
$$

\section{Number of Effective Samples for Continuity}

It was established in II.D that $n_{e s, f a}\left(n_{d}\right)<360$. In this section a higher fidelity approach is taken using the GMP, as described in III.B. $P_{f a}$ is heavily dependent on the test statistic autocorrelation function $R(\Delta t)$. An analytical upper bound was derived in [4]:

$P_{f a}\left(n, K_{f a}\right)=1-\left[1-2 \Phi\left(-K_{f a}\right)\right]\left\{1-\frac{P_{\Delta}\left(K_{f a}\right)}{1-2 \Phi\left(-K_{f a}\right)}\right\}^{n-1}$

$$
P_{\Delta}\left(K_{f a}\right) \triangleq \frac{1}{\pi} \exp \left(-\frac{K_{f a}^{2}}{2}\right) \operatorname{acos}\left[\frac{R(\Delta t)}{R(0)}\right]
$$

where $K_{f a}$ is the normalized monitor detection threshold and $\Phi(x)$ is the standard cumulative normal distribution function.

In this $P_{f a}$ analysis, we use realistic autocorrelation models for the various test statistic error contributions. Because $P_{f a}$ 
contributes only to continuity risk (not integrity), we use "average" (not worst-case) error models (described in Appendix D). A summary of results for $T_{a}=10 \mathrm{sec}$ (applicable to RNP 0.1 and 0.3 ) is provided in Table III, which leads to $(\Delta t=10 \mathrm{~s})=0.9902$, or $\beta \cong 0.001$ or $\tau_{\text {tot }} \cong 1000 \mathrm{~s}$.

TABLE III. AVERAGE AUTOCORRELATION FUNCTION VALUES (INCLUDING RATIO FOR $T_{a}=10 \mathrm{SEC}$ )

\begin{tabular}{|c|c|c|c|c|}
\hline Avg. Vals & SIS & TROPO & USER & TOTAL \\
\hline$R(0)\left[m^{2}\right]$ & 0.25 & 0.22 & 0.13 & 0.43 \\
\hline $\begin{array}{c}\alpha(\Delta t= \\
10 s)\end{array}$ & 0.9987 & 0.9945 & 0.9724 & 0.9902 \\
\hline$\beta$ & $1.3 \mathrm{e}-4$ & $5.5 \mathrm{e}-4$ & $2.8 \mathrm{e}-3$ & $9.8 \mathrm{e}-4$ \\
\hline$\tau$ & 7700 & 1800 & 360 & 1000 \\
\hline
\end{tabular}

Figure 10 shows false alert probability as a function of the normalized detection threshold for RNP $0.1 \& 0.3$, where $T_{e}=1$ hour $(n=360)$. The blue curve shows $P_{f a}$ for a single test over the exposure time, and the red curve shows the result assuming 360 independent tests. The black curves show the results using equation (2) (solid line) and direct Monte Carlo simulation (dotted line). Setting the normalized threshold at 5.33, $\left(P_{f a}=\right.$ 10-7 for a single test), leads to a false alarm probability of $\cong 10-5$ over one hour. To achieve $P_{f a}=10-7$ over the entire hour requires a threshold to 6.15 . Figures 11 and 12 show these results in terms of the number of effectively independent samples for continuity over $T_{e}=3600 \mathrm{~s}$ with $T_{a}=10 \mathrm{~s}$ for $\mathrm{RNP}$, and over $T_{e}=15 \mathrm{~s}$ with $T_{a}=6 \mathrm{~s}$ for LPV.

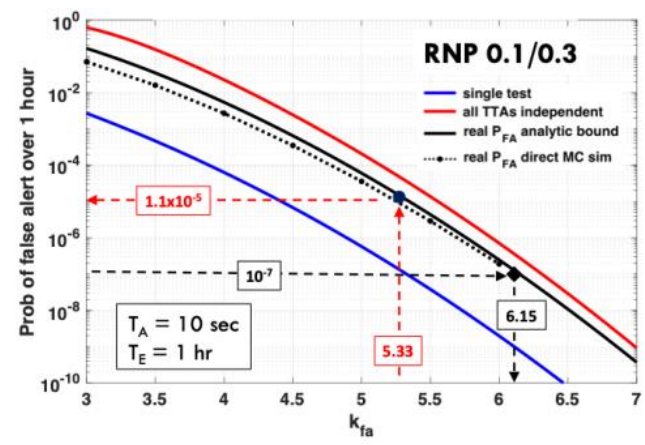

Figure 10: Probability of false alert for RNP 0.1/0.3 versus normalized threshold using 'average' GMP

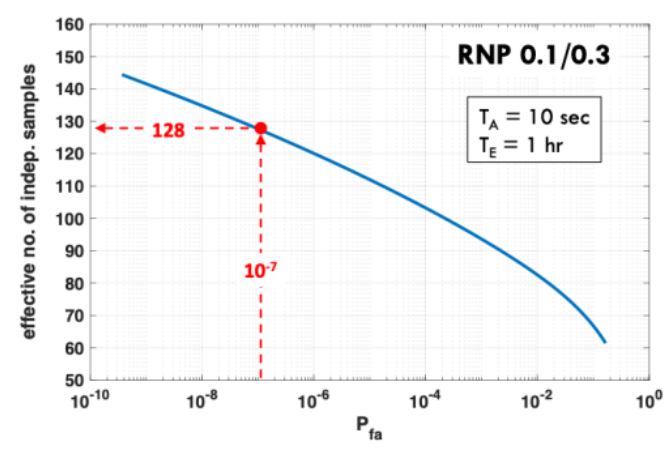

Figure 61: $n_{e s}$ for continuity vs. $P_{f a}$ for RNP 0.1/0.3 using 'average' GMP

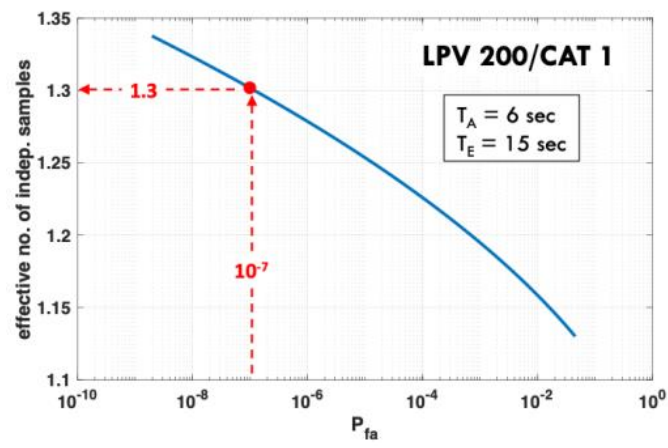

Figure 7: $n_{e s}$ for continuity vs. $P_{f a}$ for LPV 200 using 'average' GMP

\section{Effective Number of Samples for Integrity}

In this section more accurate bounds on the $n_{e s}$ for monitored modes are computed; firstly for a class of algorithms which include the baseline algorithm [5] in section III.D.1., and secondly for approaches which leverage tighter bounds on the impact of a fault in III.D.2.

1) Single GMP Algorithm (Triangle Inequality)

One approach to bounding the PHMI within the ARAIM framework is outlined in the ARAIM ADD [5]. The PHMI for this approach is bounded using the triangle inequality and is expressed by the probability of the following condition:

$$
\frac{\left|\hat{x}_{c}^{(k)}-x_{c}\right|}{\sigma_{c}^{(k)}}>K_{m d, c, k}
$$

The left-hand side is the fault-tolerant subset position solution error $\hat{x}_{c}^{(k)}-x_{c}$ (for position coordinate $c$ ), normalised by its standard deviation $\sigma_{c}^{(k)}$, under hypothesis $H_{k}$ whilst the righthand side is the Gaussian integrity factor relating to the missed detection probability allocated to the respective fault hypothesis $k$. Note that the subset position error bias is omitted from the above relations for clarity. As established above in section III.B, this quantity may be conservatively assumed to follow a Gauss Markov Process with unit variance and time constant $\tau$. The right-hand side varies as a function of the prior probability of the fault hypothesis $p_{\text {fault }, k}$, the computed protection level $P L_{c}$ and risk allocation. Employing the definition for $n_{e s}$ given in Section II.C leads to the following, with $\hat{x}_{c, i}^{(k)}$ the fault tolerant subset position error at epoch $i$ within the exposure interval of duration $T_{e}$ :

$$
n_{e s, h m i}=\frac{P\left(\cup_{j=0}^{n_{d}-n_{d a}} \cap_{i=j}^{i=j+n_{d}} d a \frac{\widehat{x}_{q, i}^{(k)}-x}{\sigma_{q}^{(k)}}>K_{m d, c, k}\right)}{P\left(\frac{\widehat{x}_{q, 0}^{(k)}-x}{\sigma_{q}^{(k)}}>K_{m d, c, k}\right)}
$$

Using the GMP model, Figure 13 shows the obtained results. 


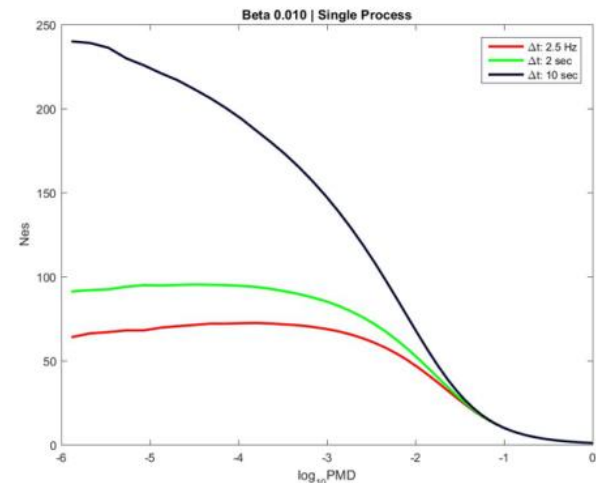

Figure 8 - Single GMP $n_{e s, h m i}(\beta=0.01$ i.e. $\tau=100 \mathrm{~s})$

TABLE IV. $\quad \operatorname{MAX} n_{e s, h m i}$ RESULTS FOR RNP 0.1/0.3

\begin{tabular}{|c|c|c|c|}
\hline & Black & Green & Red \\
\hline$n_{d}$ & 360 & 1800 & 9000 \\
\hline$n_{s}$ & 360 & 1800 & 9000 \\
\hline $\begin{array}{c}\operatorname{Max}\left(n_{e s}\right) \\
\beta=0.001: 0.01\end{array}$ & 250 & 100 & 75 \\
\hline
\end{tabular}

Table IV shows that when employing the baseline algorithm as described in the ADD [5] (green curve), a maximum $n_{e s, h m i}$ of 100 is to be expected. If on the other hand the implementation only meets the minimum requirement on fault detection frequency, a maximum of 250 is obtained. In Figures 14 and 15 results are shown for RNP and LPV-200.

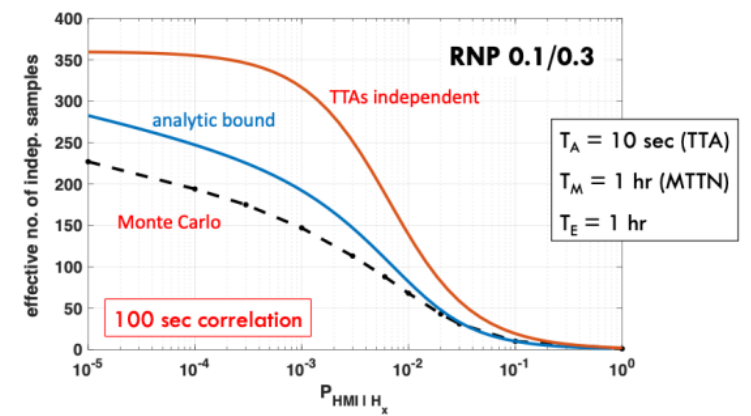

Figure 9: Number of effectively independent samples for integrity vs. $P_{h m i \mid H_{i}}$ for RNP 0.1/0.3

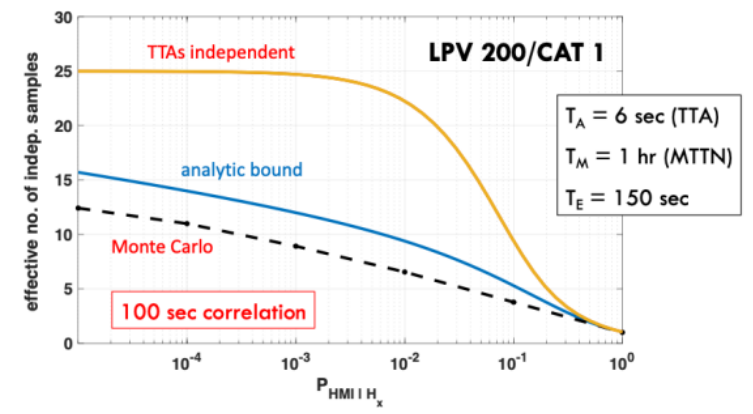

Figure 10: Number of effectively independent samples for integrity vs. $P_{h m i \mid H_{i}}$ for LPV 200

\section{2) Dual GMP Algorithm (Slope-Based Integrity)}

In this section, an alternative class of algorithm implementations are analysed. Under this class, the protection level computation uses a missed detection probability based on the test statistic and position error variables [13], [14]. The positioning failure condition and the no detection condition are then used to define an integrity event.

$$
\begin{aligned}
& \frac{\left|\hat{x}_{c}^{(0)}-x_{c}\right|}{\sigma_{c}^{(0)}}>L_{c} \\
& \frac{\left|\hat{x}_{c}^{(k)}-\hat{x}_{c}^{(0)}\right|}{\sigma_{s s, c}^{(k)}}<K_{f d, c, k}
\end{aligned}
$$

The left-hand side of (26) is the normalised position error of the full-set solution, whilst the right side is the normalised protection level, $L_{c}=P L_{c} / \sigma_{c}^{(0)}$ for coordinate $c$. The left-hand side of (27) is the normalised test statistic, for example the solution separation test statistic as defined in the baseline algorithm, whilst the right-hard side is the Gaussian k-factor relating to the allocated false detection probability to fault mode $k$. Under this approach the impact of a fault vector $\mu_{k}$ is given as follows:

$$
\begin{aligned}
& \mu_{c}^{(0)}=e_{c}^{T} S^{(0)} \mu_{k} \\
& \mu_{s s, c}=e_{c}^{T}\left(S^{(k)}-S^{(0)}\right) \mu_{k}
\end{aligned}
$$

where $S^{(0)}$ and $S^{(k)}$ respectively are the full-set and subset estimator matrices, and $e_{c}$ is a column vector of zeros except for unity at element $c$ to extract the $c$-th state. The slope, defined as ratio of $\mu_{c}^{(0)}$ and $\mu_{s s, c}$ is then a constant (not dependent on fault magnitude) [13].

$$
\text { slope }_{c}=\frac{\mu_{c}^{(0)}}{\mu_{s s, c}}
$$

The method for calculating $n_{e s, h m i}$ for these algorithms is given in further detail in [7], [14] leading to:

$$
n_{e s, h m i}=\frac{P\left(\cup_{j=0}^{n_{S}-n_{s} a} \cap_{i=j}^{i=j+n_{S} a}\left(\frac{\left|\widehat{x}_{c, i}^{(0)}\right|}{\sigma_{c}^{(0)}}>L_{c} \cap \frac{\left|\hat{x}_{c, i}^{(k)}-\widehat{x}_{c, i}^{(0)}\right|}{\sigma_{s s, c}^{(k)}}<K_{f d, c, k}\right)\right)}{P\left(\frac{\left|\hat{x}_{c, 0}^{(0)}\right|}{\sigma_{c}^{(0)}}>L_{c} \cap \frac{\left|\hat{x}_{c, 0}^{(k)}-\widehat{x}_{c, 0}^{(0)}\right|}{\sigma_{s s, c}^{(k)}}<K_{f d, c, k}\right)}
$$

The results shown in Figure are comparable to Figure such that the bounding method has little impact of on the $n_{e s, h m i}$ although the denominator in (31) is significantly smaller.

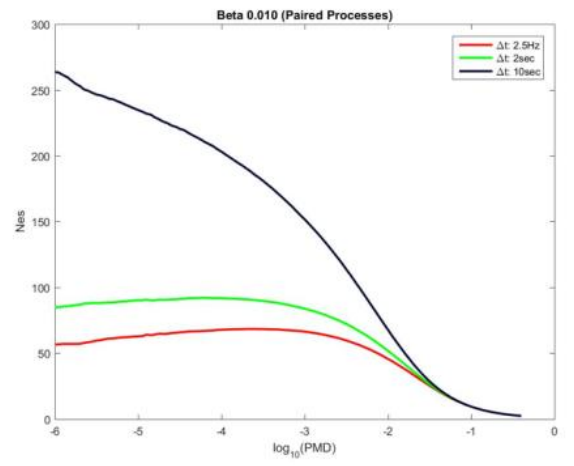

Figure 11: Dual GMP $n_{e s, h m i}(\beta=0.01)$ 


\section{E. Observed Temporal Correlations}

We evaluated the temporal correlation of the position errors observed in 23 flights ( 72 hours) collected by an FAA Global 5000 between 08/2017 and 08/2018. The data was decimated at $1 \mathrm{~Hz}$. The reference position was computed using a Precise Point Positioning algorithm (with forward backward filter) using RTKLIB tools and GPS + GLONASS L1-L2. We expect the accuracy of the position at the decimeter level. Figure shows the vertical position error and its standard deviation for one of the flights.

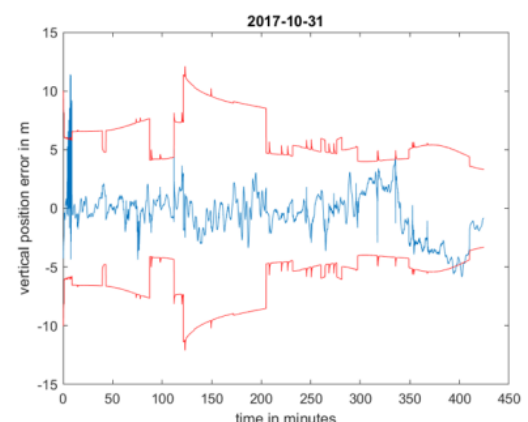

Figure 12. VPE for a flight conducted on October 31, 2017.

We then computed two empirical probability distributions: the instantaneous probability of exceeding a given limit, and the probability of exceeding the limit over an exposure interval. The ratio of these two probabilities $n_{e s, h m i}$ in Figure . The empirical curve is consistent with that in Figure 14.

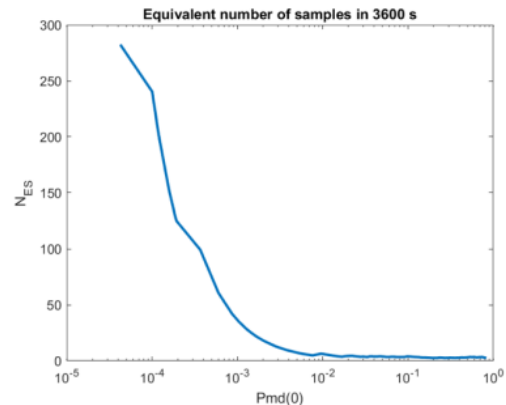

Figure 13. Empirical number of effective samples as measured over 23 flights.

\section{IMPACT ANALYSIS}

In this part, the impact of the analysis on ADD and MOPS development is described.

\section{A. Reference ARAIM ADD and MOPS}

We will update the H-ARAIM reference algorithm using the parameters $n_{e s, h m i}$ and $n_{e s, f a}$, and the upper bound given in Sections II or III. For the monitored hypotheses, we will inflate the probabilities by $n_{e s, h m i}$ and $n_{e s, f a}$ respectively for the HMI and FA probabilities. Using the notations in [2], the containment used for the test thresholds will be now given by:

$$
K_{\text {fa,east }}=K_{\text {fa,north }}=Q^{-1}\left(\frac{P_{\text {fa_hor }}}{4 N_{\text {fault modes }} n_{\text {es }, f a}}\right)
$$

$$
\begin{aligned}
& 2 \bar{Q}\left(\frac{P L_{c}-b_{c}^{(0)}}{\sigma_{c}^{(0)}}\right)+\sum_{k=1}^{N_{\text {fault modes }}} P_{\text {fault }, k} \bar{Q}\left(\frac{P L_{c}-T_{k, 3}-b_{c}^{(k)}}{\sigma_{c}^{(k)}}\right)= \\
& \frac{P H M I_{c}}{n_{e s, h m i}}\left(1-\frac{P_{\text {fault not monitored }}}{P_{V E R T}+P_{\text {HOR }}}\right)
\end{aligned}
$$

The computation of $P_{\text {fault not monitored }}$ is modified by using the probabilities of having a primary fault in the exposure time instead of the state probabilities. The probability of a primary event $P_{\text {event, } k}\left(T_{e}\right)$ (satellite or constellation fault) happening in the exposure time is given by:

$$
P_{\text {event }, k}\left(T_{e}\right)=r_{k} T_{m, k}\left(1+\frac{T_{e}}{T_{m, k}}\right)
$$

Once $P_{\text {sat }}$ and $P_{\text {const }}$ are replaced with this expression, the method to compute $P_{\text {fault not monitored }}$ remains unchanged. More details on these proposed updates can be found in [5]. The default values for $n_{e s, h m i}$ and $n_{e s, f a}$ will be 360 .

\section{B. Performance Simulations}

In order to assess the impact of this approach on ARAIM performance, we evaluated H-ARAIM coverage using both the reference ADD [5] and the modification proposed in this paper. The following settings were used:

- User: 24 h every 300s with a 10-by-10 degree grid

- $\quad$ Receiver: $\mathrm{PHMI}=10-7 / \mathrm{h}, \mathrm{PFA}=5 \times 10_{-7} / \mathrm{h}$

- Constellations: Almanacs from [2] and [3] for dual frequency GPS and Galileo

The ISM parameters were set as specified in Table V.

H-ARAIM performance is given in Table VI in terms of coverage of $99.5 \%$ availability [5]. For the degraded scenario 23-23, the drop in performance is significant. But, for other scenarios including the nominal 24-24 scenario, the modification proposed in this paper maintains a high coverage performance while properly accounting for risks over the exposure period.

TABLE V. ISM PARAMETERS USED IN COVERAGE SIMULATIONS

\begin{tabular}{|c|l|l|l|l|}
\hline Parameter & Definition & GPS & Galileo & Unit \\
\hline$r_{\text {sat }}$ & Constellation fault rate & $1 \mathrm{e}-8$ & $1 \mathrm{e}-4$ & $1 /$ hour \\
\hline$r_{\text {const }}$ & Satellite fault rate & $1 \mathrm{e}-5$ & $1 \mathrm{e}-5$ & $1 /$ hour \\
\hline MTTN & $\begin{array}{l}\text { Mean-Time-To-Notify user of } \\
\text { fault }\end{array}$ & 1 & 1 & hours \\
\hline$\sigma_{U R A}$ & Scale factor for URA & 1 & 1 & - \\
\hline$\sigma_{U R E}$ & Scale factor for URA & 1 & 1 & - \\
\hline$b_{n o m 0}$ & Additive nominal bias bound & $\begin{array}{l}0.75 \\
(\mathrm{DF})\end{array}$ & $\begin{array}{l}0.75 \\
(\mathrm{DF})\end{array}$ & meters \\
\hline$\gamma_{\text {nom }}$ & Scale factor for bias & $0(\mathrm{SF})$ & $0(\mathrm{SF})$ & \\
\hline
\end{tabular}

The equation defining the PL for coordinate $c$ will be: 
TABLE VI. EFFECT OF PROPOSED MODIFICATIONS ON COVERAGE (DEFINED AS THE PERCENTAGE OF USERS WITH 100\% AVAILABILITY) FOR 6 SCENARIOS

\begin{tabular}{|l|l|l|l|l|}
\hline $\begin{array}{l}\text { Constellation } \\
\text { configuration }\end{array}$ & \multicolumn{2}{|l|}{ URA =1 m } & URA = 2.4 m \\
\hline Approach & $\begin{array}{l}\text { ARAIM } \\
\text { ADD } \\
\text { v3 }\end{array}$ & $\begin{array}{l}\text { Proposed } \\
\text { modifications }\end{array}$ & $\begin{array}{l}\text { ARAIM } \\
\text { ADD } \\
\text { v3 }\end{array}$ & $\begin{array}{l}\text { Proposed } \\
\text { modifications }\end{array}$ \\
\hline GPS 24 & $48.0 \%$ & $39.48 \%$ & $33.37 \%$ & $20.26 \%$ \\
\hline GPS 27 & $79.5 \%$ & $71.62 \%$ & $65.81 \%$ & $53.29 \%$ \\
\hline GPS 23-Galileo 23 & $90.08 \%$ & $51.11 \%$ & $83.48 \%$ & $44.38 \%$ \\
\hline GPS 24-Galileo 24 & $98.85 \%$ & $96.37 \%$ & $97.56 \%$ & $94.39 \%$ \\
\hline GPS 27-Galileo 27 & $99.94 \%$ & $99.2 \%$ & $99.94 \%$ & $98.88 \%$ \\
\hline
\end{tabular}

\section{CONCLUSION}

This paper has evaluated the mapping of performance requirements for continuity and integrity at the operational level to the ARAIM algorithm level. It is recommended that the number of effectively independent samples be used at the algorithm level to correctly ensure requirements are met. Existing requirements have been interpreted as minimum requirements in this work to avoid unnecessary additional restrictions [11]. Strict upper bounds of 360 for RNP and 25 for LPV have been derived under that approach, along with techniques to determine lower values under certain assumptions and receiver implementations. Further reductions may be feasible with increased restrictions on the assumed model or algorithm choices.

\section{REFERENCES}

[1] FAA, "Phase II of the FAA GPS Evolutionary Architecture Study" 2010. [2] WG-C, "EU-US Cooperation on Satellite Navigation Working Group C ARAIM Technical Subgroup Milestone Report 2.” Feb-2015.

[3] WG-C, "EU-US Cooperation on Satellite Navigation Working Group C ARAIM Technical Subgroup Milestone Report 3.” Feb-2016.

[4] Pervan, Boris, Khanafseh, Samer, Patel, Jaymin, "Test Statistic Auto- and Cross-correlation Effects on Monitor False Alert and Missed Detection Probabilities," Proceedings of the 2017 International Technical Meeting of

The Institute of Navigation, Monterey, California, January 2017, pp. 562-590.

[5] WG-C ARAIM TSG, "WG-C ARAIM TSG Reference Airborne Algorithm Description Document (ADD)." 20-Jun-2019.

[6] Y. Zhai, "Ensuring Navigation Integrity and Continuity using MultiConstellation GNSS," PhD Thesis, IIT

[7] E. Bang, C. Milner, C. Macabiau, and P. Estival, "ARAIM Temporal Correlation Effect on PHMI," presented at the 31st International Technical Meeting of The Satellite Division of the Institute of Navigation (ION GNSS+ 2018), Miami, Florida, 2018, pp. 2682-2694.

[8] ICAO, "Annex 10: Aeronautical Communications" 2018

[9] Department of Defense, "Global Positioning System Standard Positioning Service Performance Standard.” 2020.

[10] European Union / European Commission / GSA, "Galileo Open Service Service Definition Document (SDD).” 2019

[11] RTCA, "Mininum Operational Performance Standards for Global Positioning System/Wide Area augmentation System Airborne Equipment DO-229D." 2006.

[12] J. Blanch et al., "Baseline Advanced RAIM User Algorithm and Possible Improvements," IEEE Trans Aerosp. Electron. Syst., vol. 51, no. 1, pp. 713 732, Jan. 2015.

[13] M. Joerger, F.-C. Chan, and B. Pervan, "Solution Separation Versus Residual-Based RAIM: Solution Separation Versus Residual-Based RAIM," Navigation, vol. 61, no. 4, pp. 273-291, Dec. 2014.
[14] E. Bang, C. Milner, C. Macabiau, and P. Estival, "Sample Temporal Correlation Effect on PHMI," p. 15.

[15] S. Perea Diaz, "Design of an integrity support message for offline advanced RAIM," 2019.

\section{Appendix A: Markov Chain Derivation for Phmi}

The joint probability of $H M I \cap H_{k}$ at any time during the exposure interval can be computed using a Markov chain with three states: (0) nominal/fault-free, (1) $H_{k}$, and (2) $H M I \cap H_{k}$. The state transitions over any time interval $T_{a}$ are modeled in Figure A.1. Transition probabilities from (0) to (1) and conversely are governed by the rate of failure and rate of CSP notification respectively. The transition probability from (1) to (2) is assumed to be independent of time as discussed for equation (B-5).

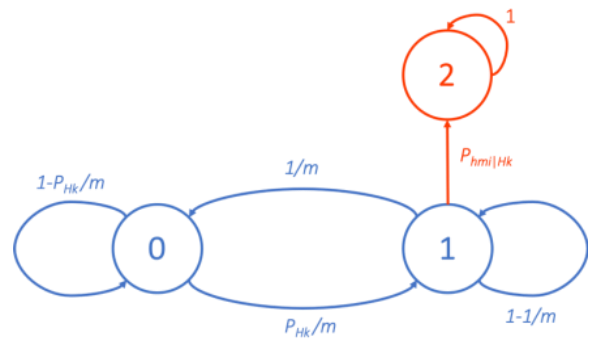

Figure A.1: Three-state Markov chain model of ARAIM monitor

To simplify notation, we recall the definitions $\bar{P}_{H_{k}} \triangleq r_{H_{k}} T_{m, k}$ and $m \triangleq T_{m, k} / T_{a}$. At any time $i$, the probability transition equations for $P_{1}(i)$ and $P_{2}(i)$ are:

$$
\begin{gathered}
P_{1}(i+1)=\frac{\bar{P}_{H_{i}}}{M} P_{0}(i)+\left(1-\frac{T_{a}}{T_{m, k}}-P_{h m i \mid H_{k}}\right) P_{1}(i) \\
P_{2}(i+1)=P_{h m i \mid H_{k}} P_{1}(i)+P_{2}(i)
\end{gathered}
$$

$P_{0}(i)$ can be eliminated using the constraint, $P_{0}(i)=1-$ $P_{1}(i)-P_{2}(i)$. To further lighten notation, define $Q \triangleq P_{h m i \mid H_{k}}$, $R \triangleq \frac{\bar{P}_{H_{k}}}{m}, S \triangleq \frac{m-1}{m}-Q-R$. Then, from (A.1) and (A.2) we have

$$
\left[\begin{array}{l}
P_{1} \\
P_{2}
\end{array}\right]_{i+1}=\left[\begin{array}{cc}
S & -R \\
Q & 1
\end{array}\right]\left[\begin{array}{l}
P_{1} \\
P_{2}
\end{array}\right]_{i}+\left[\begin{array}{c}
R \\
0
\end{array}\right]
$$

where, by definition, $P_{2}(i)=P_{h m i n H_{k}}(i)$. 


$$
P_{h m i \mid H_{k}}\left(\mu \mid H_{k}(i)\right)=P_{h m i \mid H_{k}}\left(1-P_{h m i \mid H_{k}}\right)^{\mu-i}
$$

Define $P_{H_{k}}(i)$ to be the probability of onset of $H_{k}$ between TTA intervals $i-1$ and $i$. The event itself is denoted $H_{k}(i)$. Then we can write

$$
\begin{gathered}
P_{h m i \cap H_{k}}(n)=P\left\{\bigcup_{\mu=1}^{n}\left(H M I_{\mu}, H_{k}\right)\right\}= \\
\sum_{i=1}^{n} P\left\{\bigcup_{\mu=i}^{n} H M I_{\mu} \mid H_{k}(i)\right\} P_{H_{k}}(i)
\end{gathered}
$$

At start of exposure, $P_{H_{k}}(1)=\bar{P}_{H_{k}} /\left(1+\bar{P}_{H_{k}}\right)$, which for small $\bar{P}_{H_{k}}$, means that $P_{H_{k}}(1) \approx \bar{P}_{H_{k}}$. At any time $\mu$, given a fault $H_{k}$ with onset time $i, H M I$ will be the result of two independent events: $a$ ) the CSP fails to detect and notify before time $\mu$ given a fault with onset time $i$, and $b$ ) the fault becomes hazardous at time $\mu$ and ARAIM fails to detect. We denote the probabilities of these two events as $P_{C S P \mid H_{k}}\left(\mu \mid H_{k}(i)\right)$ and $P_{h m i \mid H_{k}}\left(\mu \mid H_{k}(i)\right)$, respectively. Using mutual exclusivity of the conditional HMI onset times in the exposure interval, we can write (B.1) as:

$P_{h m i n H_{k}}(n)=\sum_{i=1}^{n} \sum_{\mu=i}^{n} P_{C S P \mid H_{k}}\left(\mu \mid H_{k}(i)\right) P_{h m i \mid H_{k}}\left(\mu \mid H_{k}(i)\right) P_{H_{k}}(i)$

(B.2)

$P_{C S P \mid H_{k}}(\mu \mid i)=\exp \left(-\frac{\mu-i}{m}\right)$

Using an exponential model and since the conditional $H M I$ onset times are mutually exclusive, using chain rule:

$$
\begin{aligned}
& P_{h m i \mid H_{k}}\left(\mu \mid H_{k}(i)\right) \\
& =P_{h m i \mid H_{k}}\left(\mu, \overline{\mu-1}, \overline{\mu-2}, \ldots, \bar{\imath} \mid H_{k}(i)\right)
\end{aligned}
$$

$=P_{h m i \mid H_{k}}\left(\mu \mid \overline{\mu-1}, \overline{\mu-2}, \ldots, \bar{l}, H_{k}(i)\right) P_{h m i \mid H_{k}}\left(\overline{\mu-1} \mid \overline{\mu-2}, \ldots, \bar{l}, H_{k}(i)\right)$

$$
\cdots P_{h m i \mid H_{k}}\left(\overline{l+1} \mid \bar{l}, H_{k}(i)\right) P_{h m i \mid H_{k}}\left(\bar{l} \mid H_{k}(i)\right)
$$

Assuming that the conditional probability of $H M I$ onset $P_{h m i \mid H_{k}}$ is the same for any TTA interval, we obtain: where $\left(1-P_{h m i \mid H_{k}}\right)^{\mu-i}$ is the probability that $H M I$ onset did not occur before time $\mu$ given fault onset at time $i$. This relation is exact if the conditional $H M I$ events are independent over TTA intervals and conservative if positively correlated, as is to be expected. Substituting (B.3) and (B.5) into (B.2) it follows that:

$$
\begin{array}{r}
P_{h m i \cap H_{k}}(n)=\sum_{i=1}^{n} \sum_{\mu=i}^{n} \exp \left(-\frac{\mu-i}{m}\right) P_{h m i \mid H_{k}}(1- \\
\left.P_{h m i \mid H_{k}}\right)^{\mu-i} P_{H_{k}}(i)
\end{array}
$$

To simplify notation, define $\alpha \triangleq \exp \left(-\frac{1}{m}\right)\left(1-P_{h m i \mid H_{k}}\right) \approx$ $\frac{m-1}{m}\left(1-P_{h m i \mid H_{k}}\right)$

$$
P_{h m i \mid H_{k}}\left\{\sum_{\mu=1}^{n} \alpha^{\mu-1} P_{H_{k}}(1)+\sum_{i=2}^{n} \sum_{\mu=i}^{n} \alpha^{\mu-i} P_{H_{k}}(i)\right\}
$$

Noting that $P_{H_{k}}(i)=\left(T_{a} / T_{m}\right) P_{H_{k}}(1)=P_{H_{k}}(1) / m$, we have

$$
\begin{array}{r}
P_{h m i n H_{k}}(n)= \\
P_{h m i \mid H_{k}} P_{H_{k}}(1)\left\{\sum_{\mu=1}^{n} \alpha^{\mu-1}+\frac{1}{m} \sum_{i=2}^{n} \sum_{\mu=i}^{n} \alpha^{\mu-i}\right\}
\end{array}
$$

To simplify further, we exploit the formula for the sum of a geometric series,

$\sum_{\ell=1}^{L} \alpha^{\ell-1}=\frac{1-\alpha^{L}}{1-\alpha}$

Then, after some algebra, (B.9) becomes

$$
\begin{array}{r}
P_{\text {minH H }_{k}}(n)=\frac{1}{1-\alpha}\left\{\left(1-\alpha^{n}\right)[1-\right. \\
\left.\left.\frac{1}{m(1-\alpha)}\right]+\frac{n}{m}\right\} P_{h m i n H_{k}}(1)
\end{array}
$$

\section{Appendix C: Derivations for Composite Faults}

As shown in Appendix B, the number of effective samples is a function of the mean time to detect. For simultaneous faults, it 
is more adequate to label this quantity the mean fault duration, since the ground segment might not necessarily detect the composite fault, but by notifying one of the faults forming the composite fault, it will stop the composite fault.

Let us consider a composite fault $k$ composed of the primary faults $k_{1}, \ldots, k_{q}$. We show that the mean time to detect $T_{m, k}$ of this is given by:

$$
T_{m, k}=\left(\sum_{v=1}^{q} \frac{1}{T_{m, k_{v}}}\right)^{-1}
$$

The fault rate corresponding to this composite fault is given by:

$$
r_{k}=p_{\text {fault }, k} \sum_{v=1}^{q} \frac{1}{T_{m, k_{v}}}
$$

where $p_{\text {fault }, k}$ is the state probability of the composite fault (and is specified in [5]). A consequence of this formula is that the probability of fault $k$ in an interval of length $T$ is given by:

$$
p_{\text {fault }, k}(T)=\left(1+T \sum_{v=1}^{q} \frac{1}{T_{m, k_{v}}}\right) p_{\text {fault }, k}
$$

In case all $T_{m} \mathrm{~s}$ are identical (which is what we expect). These formulas become:

$$
\begin{aligned}
& T_{m, k}=\frac{T_{m}}{q} \\
& r_{k}=p_{\text {fault }, k} \frac{q}{T_{m}} \\
& p_{\text {fault }, k}(T)=\left(1+\frac{q T}{T_{m}}\right) p_{\text {fault }, k}
\end{aligned}
$$

Proof:

Let us consider two events 1 and 2 , with rates $r_{1}$ and $r_{1}$ and mean time to notify $T_{m, 1}$ and $T_{m, 1}$. In order to determine the rate of the combined fault, let us consider the probability that the fault appears in an interval $\Delta t$. We define $P_{k}$ as the probability that event $k$ is present at a given time. We have the relationship:

$$
P_{k}=r_{k} T_{m, k}
$$

There can be three mechanisms for the composite fault to appear:

- fault 1 was already present and 2 starts in the infinitesimal interval $\Delta t$, or

- fault 2 was already present and 1 starts in the infinitesimal interval $\Delta t$

- $\quad$ both faults 1 or 2 appear in the interval $\Delta t$

The probability of 1 and 2 first occurring simultaneously in the interval $\Delta t$ is therefore:

$P($ events $1 \& 2$ in $[0, \Delta t])=P_{1} r_{2} \Delta t+P_{2} r_{1} \Delta t+\left(r_{1} \Delta t\right)\left(r_{2} \Delta t\right)$
To obtain the rate, we divide this expression by $\Delta t$ and let it tend to 0 :

$$
r_{12}=\lim _{\Delta t \rightarrow 0} \frac{P_{1} r_{2} \Delta t+P_{2} r_{1} \Delta t+\left(r_{1} \Delta t\right)\left(r_{2} \Delta t\right)}{\Delta t}=P_{1} r_{2}+P_{2} r_{1}
$$

Using Equation (C.7), we can write:

$$
\begin{aligned}
r_{12}=P_{1} r_{2}+P_{2} r_{1} & =P_{1} P_{2} \frac{1}{T_{m, 1}}+P_{2} P_{1} \frac{1}{T_{m, 2}} \\
= & P_{2} P_{1}\left(\frac{1}{T_{m, 1}}+\frac{1}{T_{m, 2}}\right) \\
& \text { (C. } 10)
\end{aligned}
$$

To compute the mean fault duration of the composite fault $T_{m, 12}$, we consider the probability of having 1 and 2 at a given time. Using Equation (C.7) again, we have:

$$
P_{12}=r_{12} T_{m, 12}
$$

Combining Equations (C.10) and (C.11), we get:

$$
T_{m, 12}=\left(\frac{1}{T_{m, 1}}+\frac{1}{T_{m, 2}}\right)^{-1}
$$

This result is generalized to $n$ events either by induction or by rewriting Equations (C.8) and (C.9) as follows:

$$
\begin{aligned}
& P(\text { events } 1 \& \ldots \& q \text { in }[0, \Delta t]) \\
& \quad=P_{1 \ldots q \backslash q} r_{q} \Delta t+P_{1 \ldots q \backslash q-1} r_{q-1} \Delta t \\
& \quad+\cdots P_{1 \ldots q \backslash 1} r_{1} \Delta t+O\left(\Delta t^{2}\right)
\end{aligned}
$$$$
\text { (C.13) }
$$

where $P_{1 \ldots q \backslash k}$ is the state probability of all $q$ events except $k$. We have:

$$
P_{1 \ldots q \backslash k} r_{k}=P_{1 . . q} \frac{1}{T_{m, k}}=P_{1} . . P_{q} \frac{1}{T_{m, k}}
$$

As a consequence, Equation (C.10) generalizes to:

$$
r_{1 . . q}=P_{1} \ldots P_{q}\left(\frac{1}{T_{m, 1}}+\cdots+\frac{1}{T_{m, q}}\right)
$$

And the mean fault duration is given by:

$$
T_{m, 1 \ldots q}=\left(\frac{1}{T_{m, 1}}+\cdots+\frac{1}{T_{m, q}}\right)^{-1}
$$

Appendix D: Autocorrelation Model

For the continuity risk analysis, we are interested in "average," not worst-case error models. The contributors to nominal test statistic error are the following: multipath and noise at the user aircraft, satellite orbit and clock error, and residual (post correction) tropospheric error. In parts D.1, D.2 and D.3, these three sources of error are addressed. In D.4 the models are then extended to worst-case conditions

\section{D.1 User Multipath and Receiver Noise}

Data provided by the Boeing Company from flight tests conducted with a 787 aircraft was used to create average autocorrelation models for multipath and receiver noise [4]. 
Raw GPS L1 code-minus-carrier data, with ionospheric effects removed, was analyzed to produce these models. Figure D.1 shows the sample means and standard deviations binned by satellite elevation at 5 degrees of resolution. The results suggest that on average it is reasonable to assume a zero-mean distribution with a standard deviation of $\sigma_{\mathrm{U}} \approx 0.4 \mathrm{~m}$ for all elevations for raw code multipath and receiver noise.

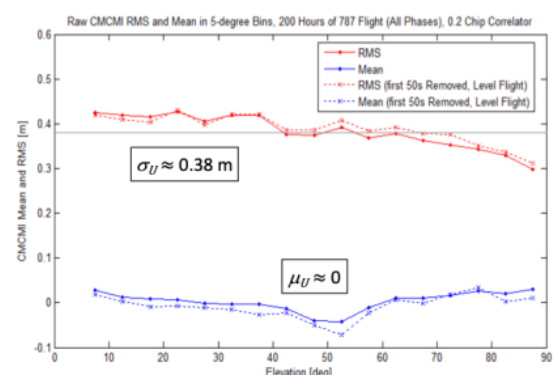

Figure D.1: Boeing 787 Flight Data: Airborne Mean and Standard Deviation

Figure D.2 shows a composite of autocorrelation traces of the flight data. A number of traces with long correlation times are clearly evident; these are caused by antenna group delay and must be accounted for in the autocorrelation model. Figure D.3 consolidates the autocorrelation data into empirical cumulative distribution functions for a number of discrete lag times. The median user-error time constant is approximately $\tau_{U} \approx 14 \mathrm{sec}$.

Raw multipath and noise is affected by carrier-smoothing of the code with filter time constant $\tau_{\mathrm{F}}$. (The typically assumed filter time constant is $\tau_{\mathrm{F}}=100 \mathrm{sec}$, but it may differ for ARAIM.) The resulting autocorrelation function for the smoothed code error is:

$$
R_{U}(t)=\kappa \sigma_{M}^{2} \frac{\tau_{U}}{\tau_{F}^{2}-\tau_{U}^{2}}\left(\tau_{F} e^{-|t| / \tau_{F}}-\tau_{U} e^{-|t| / \tau_{U}}\right)
$$

where

$$
\kappa \triangleq \frac{f_{L 1}^{4}+f_{L 5}^{4}}{\left(f_{L 1}^{2}-f_{L 5}^{2}\right)^{2}}
$$

is the error scale factor for the L1-L5 observable relative to L1.

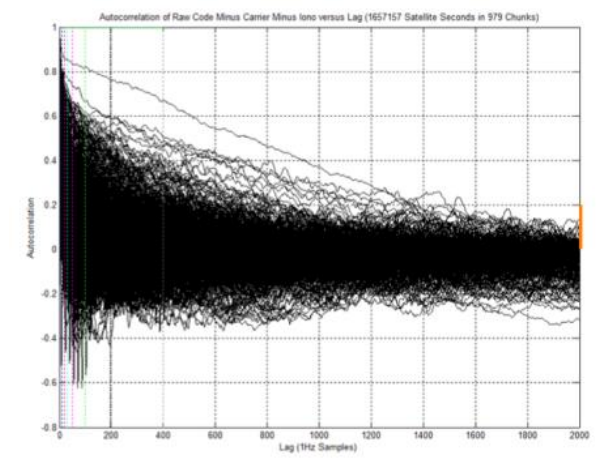

Figure D.2: Boeing 787 Flight Data Autocorrelation Traces

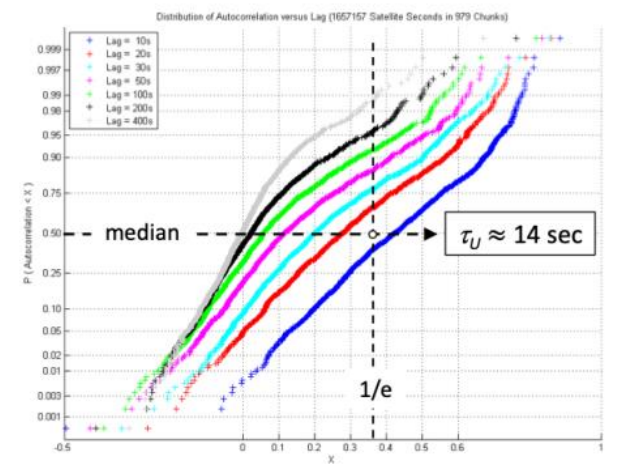

Figure D.3: Boeing 787 Data Autocorrelation by Lag Time

\section{D.2 Residual Tropospheric Error}

From DO 229E [11], Appendix R, Section R.4.1, we are instructed that, "Tropospheric error shall be modeled using a first-order Gauss-Markov process with a 30-minute correlation time $\left(\tau_{\mathrm{T}}\right)$. The sigma shall be scaled per the tropospheric residual error sigma equation defined in Appendix A, Section A.4.2.5 ... Note: The 30-minute correlation time representative of a typical storm system passing through." This equation is plotted versus satellite elevation in Figure D.4 (right). Figure D.4 (left) sows the average GPS satellite elevation at different user latitudes. The global mean (i.e., the average value of the curve) is $32.4 \mathrm{deg}$. The corresponding value at that elevation on Figure D.4 (right) is $\sigma_{\mathrm{T}} \approx 0.22 \mathrm{~m}$.
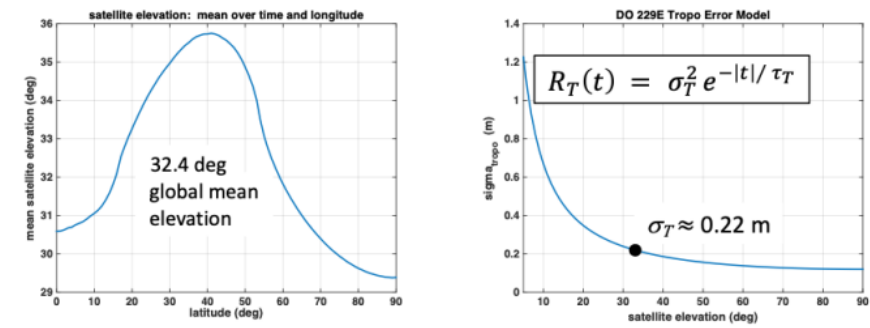

Figure D.4: Average elevations of GPS satellites (left) and residual tropospheric error vs. elevation (right)

\section{D.3 GPS Satellite Orbit and Clock Error}

To obtain average error models for satellite signal in space ranging error (SISRE), we use as proxy radial-minus-clock autocorrelation data provided by Stanford University. Example autocorrelation functions for two satellites with different clock types are shown in Figure D.5. The data was sampled at $\mathrm{T}_{S}=$ 15 min intervals, and autocorrelation ratios $R_{S}\left(T_{S}\right) / R_{S}(0)$ were obtained. The average value of this raita across GPS satellites was 0.9994. However, because of the performance variations between the Rubidium and Cesium clocks to be conservative in the example computation of the number of effectively independent samples for continuity in section 2.3, 
we use the minimum value across the satellites, $R_{S}\left(T_{S}\right)$ / $\mathrm{R}_{\mathrm{S}}(0)=0.9987$.

Figure D.6 shows the history of 95\% satellite ranging error due to satellite orbit and clock errors. The colored lines are the results for individual satellites. The heavy black curve is the average across all satellites. Currently, the average $95 \%$ error value is approximately $1 \mathrm{~m}$, so we use $\sigma_{\mathrm{S}} \approx 1 \mathrm{~m}$.

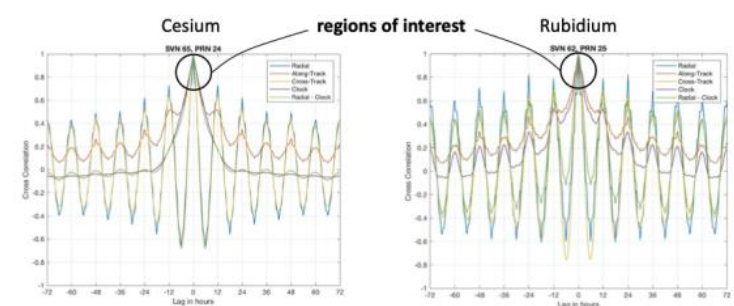

Figure D.5: Example autocorrelation functions for radialminus-clock error for two GPS satellites with different clock types

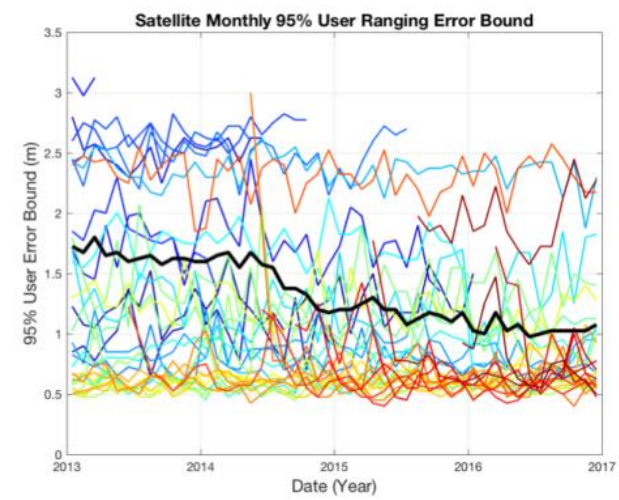

Figure D.6: Histories of $95 \%$ satellite ranging error due to satellite orbit and clock errors

TABle D.I Autocorrelation Ranges for Low, Medium AND High ELEVATION SATELLITES

\begin{tabular}{|c|c|c|c|c|c|c|}
\hline Elevation & low & med & high & low & med & High \\
\hline $\begin{array}{c}\alpha \\
(\Delta t= \\
10 s)\end{array}$ & 0.9945 & 0.9945 & 0.9950 & 0.9938 & 0.9873 & 0.9829 \\
\hline$\beta$ & $5.6 \mathrm{e}-4$ & $5.6 \mathrm{e}-4$ & $5.0 \mathrm{e}-4$ & $6.1 \mathrm{e}-4$ & $12.9 \mathrm{e}-4$ & $17.3 \mathrm{e}-4$ \\
\hline$\tau$ & 1800 & 1800 & 2000 & 1600 & 780 & 580 \\
\hline
\end{tabular}

